\title{
ANÁLISIS DE LAS TENDENCIAS DE LA PRODUCTIVIDAD DE LOS SISTEMAS DE ASERRIO DE Eucalyptus globulus Labill EN EL VALLE DEL MANTARO
}

\author{
ANALYSIS OF THE TENDENCIES OF THE PRODUCTIVITY OF THE SYSTEMS \\ OF SAWMILLS OF Eucalyptus globulus Labill IN THE VALLEY OF THE MANTARO
}

\author{
Hugo Miguel Miguel'
}

\section{RESUMEN}

La productividad de madera aserrada de eucalipto (Eucalyptus globulus Labill) en los aserraderos del valle del Mantaro siempre ha variado entre estos; encontrándose aserraderos prósperos y poco exitosos; estas diferencias percibidas han motivado a estudiar con criterio sistémico las tendencias de la productividad de madera aserrada. Los estudios se realizaron en cinco aserraderos de sierra banda y en cuatro de sierra circular. Los equipos de medición utilizados fueron: tacómetro digital, reloj comparador, cronómetro digital y vernier. Se midieron el espesor de viruta, velocidad de corte, velocidad de alimentación, paso, afilado de las sierras, aspectos ergonómicos y la productividad. Los resultados del análisis de las tendencias de la productividad están directamente asociados con el espesor de viruta, calidad de afilado de sus sierras y el trato al personal operativo. El aserradero de sierra banda que alcanzó el mejor nivel de productividad de 2,35 $\mathrm{m}^{2} /$ minuto, generó un espesor de viruta de 489 micras, con calidad de afilado "bueno" y trato "regular" al personal; contrariamente, el aserradero que alcanzó el más bajo nivel de productividad, generó un espesor de viruta de 218 micras, con calidad de afilado "deficiente" y trato "deficiente" al personal. La relación directa entre la productividad y el espesor de viruta está asociada con el manejo de las velocidades de corte y de alimentación de la madera; altas velocidades de corte generan menores espesores de viruta, desgaste acelerado de los filos de las sierras, consecuentemente baja productividad. Las relaciones encontradas se podrían utilizar para reajustar el manejo de las máquinas y herramientas de aserrío, para elevar la productividad y la rentabilidad de los aserraderos sin utilizar recursos económicos significativos.

Palabras clave: madera aserrada, productividad en aserrío, espesor de viruta.

\section{SUMMARY}

The sawed wooden productivity of eucalyptus (Eucalyptus globulus Labill) in the sawmills of the valley of the Mantaro it has always varied among these, being prosperous and not very successful sawmills; these perceived differences have motivated to study with systemic approach the tendencies of the sawed wooden productivity. Five sawmills of saw band and four of circular saws were studied. The used mensuration teams were: digital tachometer, clock comparator, digital chronometer and vernier. They were measured the chip thickness, court speed, feeding speed, step, sharp of the sawmills, ergonomic aspects and the productivity. The results of the analysis of the tendencies of the productivity are directly associated with the chip thickness, quality of sharp of their sawmills and the treatment to the operative personnel. The sawmill of band that reached the best level of productivity of 2,35 $\mathrm{m}^{2} /$ minuto, generated a thickness of chip of 489 microns, with quality of sharp "good" and I try "to regulate" to the personnel; contrarily, the sawmill that reached the lowest level of productivity, generated a thickness of chip of 218 microns, with quality of sharp "faulty" and I try "faulty" to the personnel. The direct relationship between the productivity and the chip thickness is associated with the handling of the court speeds and of feeding of the wood (1); high court speeds generate smaller chip thickness, quick waste of the edges of the mountains, consequently low productivity. The opposing relationships could be used to readjust the handling of the machines and saw tools, to elevate the productivity and the profitability of the sawmills without using significant economic resources.

Key words: sawed wood, productivity in sawmill, chip thickness.

${ }^{\prime}$ Facultad de Ciencias Forestales y del Ambiente. Universidad Nacional del Centro del Perú. 


\section{INTRODUCCIÓN}

En la actualidad en el valle del Mantaro existen más de 60 aserraderos que transforman la madera de eucalipto, produciendo aproximadamente 24 $641,60 \mathrm{~m}^{3}$ de madera aserrada al año (2). Entre estas plantas industriales existe variabilidad significativa de la productividad del corte de la madera $\left(\mathrm{m}^{2}\right.$ de madera cortada por minuto), repercutiendo directamente sobre la rentabilidad económica.

En efecto, existen aserraderos con niveles aceptables de rentabilidad, como también los hay contrariamente; estas diferencias pueden ser estudiadas cuando un aserradero es visto como un sistema dinámico y abierto, identificando las relaciones entre las variables que intervienen en el proceso de corte y al mismo tiempo analizando las salidas del sistema, como es el caso de la productividad en aserrío.

Bajo este enfoque el estudio tuvo como objetivos, analizar las tendencias de la productividad de madera aserrada incluyendo los principales elementos del proceso y sus interrelaciones entre estos con criterio sistémico. Dentro de los elementos que interactúan en la determinación de la productividad se consideraron: el espesor de viruta, la velocidad de alimentación de la materia prima, la velocidad de corte, el paso de los dientes, calidad de afilado de las sierras y los aspectos ergonómicos.

\section{MATERIAL Y METODOS}

La investigación se realizó en cuatro aserraderos de sierra circular y en cinco aserraderos de sierra banda del Valle del Mantaro de la Región Junín. Los equipos utilizados para la obtención de datos

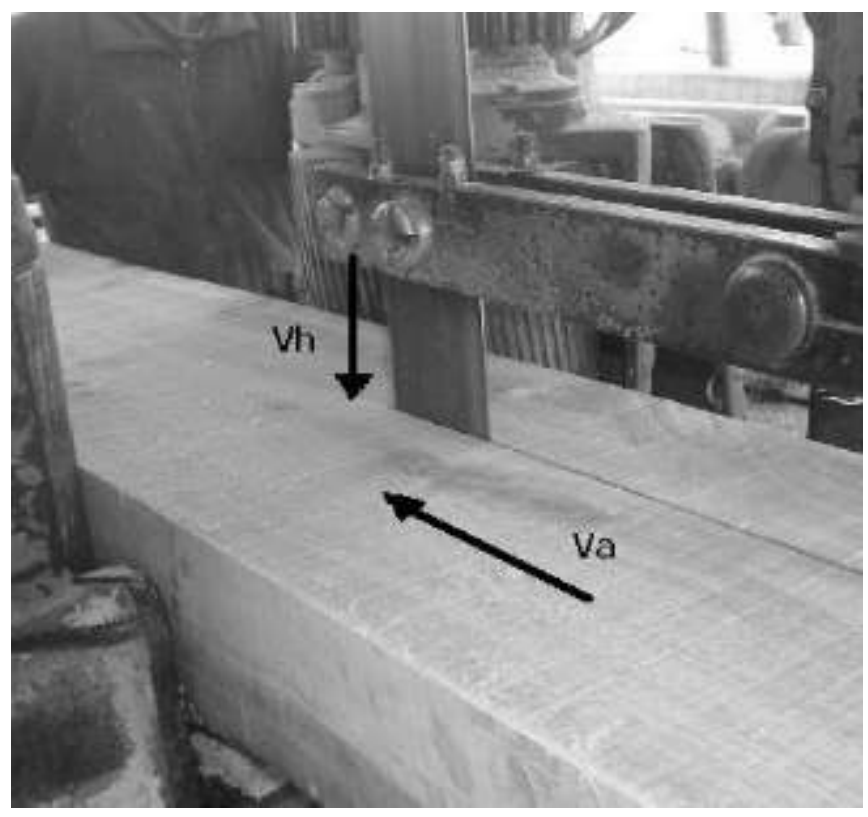

fueron: tacómetro digital, reloj comparador, wincha metálica, vernier, y cámara fotográfica y de video digital.

El método utilizado fue descriptivo y correlativo. Los métodos específicos consistieron en medir el espesor de viruta de aserrío en micras (en función de la velocidad de alimentación, velocidad de corte y el paso de las sierras) y la productividad, expresados en $\mathrm{m}^{2}$ de madera cortada por minuto (en función de la longitud de la troza, ancho de corte efectuado por la sierra en la troza y el tiempo de corte de un extremo hasta el otro de la troza).

Se evaluaron los siguientes elementos del proceso de aserrío: las características de las trozas como entrada del sistema, diámetro de las sierras, revoluciones por minuto de las sierras, paso de las sierras, velocidad de corte, velocidad de alimentación de la madera y la productividad de madera aserrada como salida del sistema; calidad del afilado de las sierras y los aspectos ergonómicos.

Para el cálculo del Espesor de Viruta se utilizó la siguiente fórmula: $\mathrm{EV}=(\mathrm{Va})(\mathrm{p}) /(\mathrm{Vh})$. Donde: $\mathrm{EV}=$ espesor de viruta (en micras); $\mathrm{Va}=$ velocidad de alimentación (en $\mathrm{m} /$ minuto); $\mathrm{Vh}=$ velocidad de corte (en $\mathrm{m} /$ minuto), y $\mathrm{p}=$ paso (en $\mathrm{mm}$ ). Y para el cálculo de la productividad de madera aserrada, la siguiente fórmula: $\mathrm{P}=(\mathrm{L})(\mathrm{A}) / \mathrm{T}$, donde: $\mathrm{P}=$ Productividad (en $\mathrm{m}^{2} /$ minuto); $\mathrm{L}=$ longitud de la troza (en $\mathrm{m}$ ); $\mathrm{A}=$ ancho de corte que se genera transversalmente al eje longitudinal de la troza (en $\mathrm{m})$, y $T=$ tiempo de corte desde un extremo hasta el otro de la troza en minutos (1). Finalmente se analizaron los resultados de las salidas de los sistemas (productividad), de los aserraderos de sierra banda y los aserraderos de sierra circular.

\section{RESULTADOS}

Durante el estudio de las tendencias de la productividad de madera aserrada en aserraderos de sierra circular y de sierra banda que cortan la madera de eucalipto en el Valle del Mantaro se han encontrado diferencias (tablas 1 y 2); en efecto, hay aserraderos que tienen niveles de productividad aceptables, contrariamente también existen aserraderos con niveles de productividad muy bajos.

Los aserraderos que tuvieron los más altos índices de productividad (Aserradero Alfa de sierra banda, con 2,35 $\mathrm{m}^{2} /$ minuto de productividad), y el (Aserradero A. Nieto de sierra circular, con 2,90 $\mathrm{m}^{2}$ /minuto de productividad), también tuvieron los valores más altos de espesor de viruta: 489 y 1073 micras respectivamente.

Contrariamente, los aserraderos que tuvieron los 


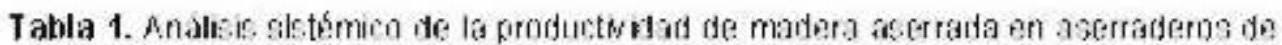
sierra toanda

\begin{tabular}{|c|c|c|c|c|}
\hline $\begin{array}{c}\text { Razón Social del } \\
\text { Asen adero }\end{array}$ & $\begin{array}{l}\text { Productividad } \\
\left(\mathrm{m}^{2} \text { /minuto }\right)\end{array}$ & $\begin{array}{c}\text { Espesor de } \\
\text { viruta } \\
\text { (micras) }\end{array}$ & $\begin{array}{l}\text { Nivel de } \\
\text { afilado de } \\
\text { las sierras }\end{array}$ & $\begin{array}{c}\text { Nived ded } \\
\text { trato al } \\
\text { personal }\end{array}$ \\
\hline ALFA & 2,35 & 489 & Bueno & Regular \\
\hline INSISA & 2,10 & 414 & Bueno & Regular \\
\hline Negociausnes Poma & 1,78 & 290 & Regular & Regular \\
\hline Aserradero Gregorio Porn s & 1,60 & 248 & Regular & Deficiente \\
\hline Assarmatyo carvoial & 1,10 & 218 & Doficipante & Deficiente \\
\hline
\end{tabular}

Tabla 2. Análisis sistemico de la productwidad de madera as grrada en aserraderas de sierr a circular

\begin{tabular}{|c|c|c|c|c|}
\hline $\begin{array}{l}\text { Razón Social del } \\
\text { Aserradero }\end{array}$ & $\begin{array}{l}\text { Productivi dad } \\
\text { (m² aninuto) }\end{array}$ & $\begin{array}{l}\text { Espesor de } \\
\text { Vinuta } \\
\text { (imicras) }\end{array}$ & $\begin{array}{l}\text { Mivel de } \\
\text { afilado de } \\
\text { las sterras }\end{array}$ & $\begin{array}{l}\text { Nivel del } \\
\text { trato al } \\
\text { personal }\end{array}$ \\
\hline A-serradiero A Nielo & 2,90 & 1073 & Eueno & Rieguar ar \\
\hline Aserradero Herrera & 2,40 & 933 & Regular & Reguar \\
\hline Áserradero oié & 1,70 & 521 & Deficierite & Regular \\
\hline Aserradero Cordero & 1,40 & 480 & Deficiente & Reguar \\
\hline
\end{tabular}

más bajos índices de productividad (Aserradero Carvajal de sierra banda, con $1,10 \mathrm{~m}^{2} /$ minuto de productividad), y el (Aserradero Cordero de sierra circular, con $1,40 \mathrm{~m}^{2} /$ minuto de productividad), también tuvieron los valores más bajos de espesor de viruta: 218 y 480 micras respectivamente.

Asimismo, se observan que estas tendencias también están asociadas con el nivel de afilado de las sierras y el nivel del trato al personal obrero de los aserraderos; en las tablas 1 y 2 , se observan que los niveles de afilado "bueno" y "regular" trato al personal obrero, están asociadas con los aserraderos de mayor productividad de madera aserrada (Aserraderos de sierra banda: ALFA e INSISA; Aserraderos de sierra circular: A. Nieto y Herrera). Contrariamente, los niveles de afilado "deficiente" y trato "deficiente" al personal obrero están relacionados con los aserraderos de menor productividad (Aserraderos de sierra banda: Gregorio Poma y Carvajal; Aserraderos de sierra circular: Oré y Cordero).

\section{DISCUSIÓN}

Las diferencias de la productividad en aserrío entre las plantas industriales que transforman mecánicamente la madera de eucalipto en el Valle del Mantaro son muy visibles, por efecto de las variaciones en las condiciones de corte, que afectan en la formación de la viruta del proceso de aserrío, particularmente del espesor de la viruta (3). En efecto, el aserradero de sierra banda con mayor productividad generó un espesor de viruta de 489 micras, como consecuencia del manejo de altas velocidades de alimentación y relativamente bajas velocidades de corte, ya que la velocidad de alimentación tiene relación directa con el espesor de viruta, mientras que la velocidad corte tiene relación inversa $(4,5)$.

Los aserraderos con los más altos valores de EV, tienen mayor capacidad de cortar la madera en menor tiempo, porque el $\mathrm{EV}$, en términos prácticos significa el avance lineal cortando la madera, por el efecto directo de la velocidad de alimentación; contrariamente, los aserraderos con los más bajos valores de EV, la productividad en aserrío es bajo porque las velocidades de alimentación son menores como efecto de esto, el nivel de avance lineal del proceso de corte es

menor (1).

Al análisis de la variable EV relacionado con la productividad en aserrío, también se añade el efecto de la calidad del afilado de los dientes cortantes de las sierras banda y sierras circulares; obviamente, los dientes mejor afilados tienen mayor capacidad de corte, contribuyendo positivamente a favor de la productividad en aserrío. Los valores más altos de EV, también evitan el desgaste acelerado de los filos de los dientes, acción indirecta que también favorece para incrementar la productividad de madera aserrada; lo que interesa es cortar la madera en el menor tiempo posible.

Asimismo, un aspecto también importante que se ha detectado es la influencia del nivel de trato al personal operativo de los aserraderos a favor o en contra de la productividad de madera aserrada; los obreros bien tratados en el sentido de alimentación adecuada, incentivos económicos y medidas de seguridad industrial tienen un efecto positivo, evitando tiempos muertos durante el proceso de aserrío, que obviamente contribuyen positivamente con la productividad.

Con los análisis sistémicos efectuados se puede concluir que los aserraderos con los más altos niveles de productividad, por lo tanto con más rentabilidad, son los que manejan mejor las condiciones de corte (mayor EV), con los mejores niveles de afilado de las sierras y los que brindan los mejores niveles de trato ergonómicos a sus obreros forestales; sin embargo conseguir altos valores de espesor de viruta también implica también utilizar 
motores con adecuadas potencias, y condiciones adecuadas de transmisión de estas, aspectos que aún faltan investigar. Existen otros factores que también influyen en la rentabilidad de los aserraderos, el rendimiento en función de las trozas a procesar, $(6,7,8)$.

\section{REFERENCIAS BIBLIOGRÁFICAS}

1. Ninin L. Manual de aserrado y afilado. Facultad de Ciencias Forestales. Mérida: Universidad Los Andes; 1985.

2. Malca O. Eucalipto. Seminario de Agro Negocios. Lima: Universidad del Pacífico; 2000.

3. Vega M. Caracterización de la rugosidad superficial y de la energía de corte durante el maquinado de Pinus radiata. Bosque. Valdivia, Chile. 2005; 26(1): 101-8.

4. Aguilera A, Vega M. Efecto del cambio de las condiciones de maquinado sobre la rugosidad superficial de Pinus radiata D. Don. BOSQUE. 2004; 25(3): 25-32.

5. Néri AC, Gonsalves R, Hernández, RE. Fuerzas de corte ortogonal 90-90 en tres especies de madera de eucalipto. Revista Brasilera de Ingeniería Agrícola y Ambiental. 2000; 4(2): 27580.

6. Martínez G, Cellini JM, Lencinas MV, Díaz B, Peri $\mathrm{PL}$, Vukasovic RF. Funciones de rendimiento volumétrico en pie y en aserradero para la lenga (Nothofagus pumilio). Ciencias Forestales. 2002; 15 (2): 32-45.

7. Quirós R, Chinchilla O, Gómez M. Rendimiento en aserrío y procesamiento primario de madera proveniente de plantaciones forestales. Agronomía Costarricense. 2005; 29(2): 7-15.

8. Quirós R, Chinchilla O, Gómez M. Consumo de madera en troza por dos aserraderos portátiles con sierra de banda horizontal. Agronomía Costarricense. 2005; 29(2): 17-25.

E-mail: hmiguel21@gmail.com 